\title{
Adjoint-based formulation for computing derivatives with respect to bed boundary positions in resistivity geophysics
}

\author{
T. Chaumont-Frelet • M. Shahriari • D. \\ Pardo
}

Received: date/ Accepted: date

\begin{abstract}
In inverse geophysical resistivity problems, it is common to optimize for specific resistivity values and bed boundary positions, as needed, for example, in geosteering applications. When using gradient-based inversion methods such as Gauss-Newton, we need to estimate the derivatives of the recorded measurements with respect to the inversion parameters. In this article, we describe an adjointbased formulation for computing the derivatives of the electromagnetic fields with respect to the bed boundary positions. The key idea to obtain this adjoint-based formulation is to separate the tangential and normal components of the field, and treat them differently. We then apply this method to a $1.5 \mathrm{D}$ borehole resistivity problem. We illustrate its accuracy and some of its convergence properties via numerical experimentation by comparing the results obtained with our proposed adjoint-based method vs. both the analytical results when available and a finite differences approximation of the derivative.
\end{abstract}

Keywords resistivity inversion, adjoint state, electromagnetic, borehole geophysics, Maxwell's equations

\footnotetext{
T. Chaumont-Frelet

Univeristé Côte d'Azur, Inria, CNRS, LJAD, France.

Basque Center for Applied Mathematics, (BCAM), Bilbao, Spain.

E-mail: theophile.chaumont@inria.fr

M. Shahriari

Basque Center for Applied Mathematics, (BCAM), Bilbao, Spain.

Mazarredo 14, E48009 Bilbao, Spain.

E-mail: m.shahriari.sh@gmail.com.com

D. Pardo

University of the Basque Country (UPV/EHU), Leioa, Spain.

Basque Center for Applied Mathematics, (BCAM), Bilbao, Spain.

Ikerbasque (Basque Foundation for Sciences), Bilbao, Spain.

E-mail: dzubiaur@gmail.com
} 


\section{Introduction}

We consider resistivity measurements to characterize the electrical properties of the subsurface. There exist: (a) on surface resistivity measurements acquisition systems such as controlled source electromagnetic (CSEM) [1] and Magnetotellurics (MT) [5, 6], and (b) borehole logging measurements such as those obtained with logging-while-drilling (LWD) devices 7 9, including the so-called deep and extra-deep [10, 11] logging devices used for geosteering purposes. Recently developed LWD resistivity measurements are able to measure all nine components of the magnetic field, namely $H_{x x}, H_{x y}, H_{x z}, H_{y x}, H_{y y}, H_{y z}, H_{z x}, H_{z y}, H_{z z}$, where the first and second sub-indexes indicate the orientation of the transmitter and the receiver, respectively.

In LWD resistivity measurements, the original Earth's subsurface model is often approximated by a sequence of 1D layered models [8. Such an approximation often provides reasonable results due to the limited depth of investigation of LWD resistivity measurements compared to the assumed thickness of the geological layers. In the presence of a 3D point source, a 1D formation model allows to reduce the dimensionality of the problem from $3 \mathrm{D}$ to the so-called $1.5 \mathrm{D}$ via a Hankel transform (or two Fourier transforms) (see, e.g., [12 14]). This 1.5D approximation can also be used to obtain an initial subsurface resistivity distribution from marine CSEM measurements [15].

Resistivity measurements are inverted to map the Earth's subsurface (see, e.g., [3, 7, 8, ). Using gradient-based inversion techniques (e.g., Gauss-Newton), we need to estimate the derivatives of the simulated measurements with respect to the inversion variables to form the Jacobian matrix. These inversion variables are often the (constant) resistivity values of specific layers and their bed boundary positions. It is well-known how to compute derivatives with respect to the (constant) resistivity values for each layer, both numerically and semi-analytically (see, e.g., [8, 14]). However, to the best of our knowledge, a fast adjoint-based formulation to compute derivatives with respect to the bed boundary positions in resistivity geophysical problems has not been published before. Such formulation employs the adjoint operator (transpose of the complex conjugate) to build an auxiliary (dual) problem whose solution facilitates the rapid construction of derivatives, as shown in 16. 17. As a result, it allows to more rapidly compute accurate approximations of the derivatives than those obtained with a traditional finite differences approach.

The main contribution of this work is to provide an adjoint-state formulation to compute derivatives of resistivity measurements with respect to the bed boundary positions and analyze its performance. The fundamental idea to obtain such formula is to treat the tangential and normal components of the field separately. To do so, we employ a technique that is presented in [18] to compute the derivative of the measurements with respect to the conductivity values. It turns out that when computing derivatives with respect to the bed boundary positions, some adaptations are required in order to derive the correct formula carefully.

In Section 2 we describe 3D Maxwell's equations, and we construct a variational formulation for the reduced wave equation. Section 3 evaluates an adjoint-state formulation to compute the derivative of a measurement with respect to a bed boundary position. We verify and analyze the main advantages and limitations of our adjoint-based method via numerical experimentations in Section 4 Conclusions are in Section 5. This article also incorporate two appendices. The first 
one shows how to compute the derivatives of the phase difference and attenuation with respect to the bed boundary positions, while the second one describes a $1.5 \mathrm{D}$ formulation for computing derivatives with respect to the bed boundary positions.

\section{Model problem}

We consider 3D Maxwell's equations to model the EM fields (see e.g., [14, 19 21]). We have:

$$
\begin{aligned}
& \nabla \times \mathbf{H}=\tilde{\boldsymbol{\sigma}} \mathbf{E}+\mathbf{J} \\
& \nabla \times \mathbf{E}=i \omega \boldsymbol{\mu} \mathbf{H}+i \omega \boldsymbol{\mu} \mathbf{M}
\end{aligned}
$$

where $\mathbf{E}$ is the complex-valued electric field, $\mathbf{H}$ is the magnetic field, $\omega=2 \pi f$ is the angular frequency, where $f>0$ is the frequency of the transmitter, and $\tilde{\boldsymbol{\sigma}}=\boldsymbol{\sigma}-i \omega \varepsilon$, where $\boldsymbol{\sigma}$ and $\varepsilon$ are the conductivity and the permittivity tensors of the medium, respectively, $i$ is the imaginary unit, and $\boldsymbol{\mu}$ is the magnetic permeability tensor of the media. $\mathbf{J}$ and $\mathbf{M}$ are the electric and magnetic volumetric current sources, respectively. For the sake of simplicity, and as it occurs in most geophysics applications (see e.g., [14, 21]), we restrict to the case where $\varepsilon=\varepsilon_{0} \mathbf{I}_{3}$ and $\boldsymbol{\mu}=\mu_{0} \mathbf{I}_{3}$ with $\mathbf{I}_{3}$ being the $3 \mathrm{D}$ identity matrix, and $\varepsilon_{0}$ and $\mu_{0}$ being the vacuum permittivity and permeability, respectively. We also assume to have transversely isotropic (TI) materials with piecewise-constant resistivity distribution, which varies only along $z$-direction. This assumption is often used in borehole resistivity inversion for geosteering purposes (see, e.g., [8, 22]). Therefore, the conductivity tensor $\boldsymbol{\sigma}$ of the formation is given by:

$$
\boldsymbol{\sigma}=\left(\begin{array}{ccc}
\sigma_{\mathbf{t}} & 0 & 0 \\
0 & \sigma_{\mathbf{t}} & 0 \\
0 & 0 & \sigma_{\mathbf{n}}
\end{array}\right),
$$

where $\sigma_{\mathbf{t}}$ and $\sigma_{\mathbf{n}}$ are strictly positive piecewise-constant functions of $z$. In (3), subscript $\mathbf{t}$ indicates the tangential $x y$-plane and $\mathbf{n}$ the normal component. We employ a similar notation for vector fields. Thus, if $\mathbf{w}$ is a vector, $\mathbf{w}_{\mathbf{t}}$ denotes the $2 \mathrm{D}$ vector that consists of its two tangential component along the $x y$-plane, and $w_{\mathbf{n}}$ is its normal component. Though it is possible to solve problem (1)-(2) directly, we often combine both equations to arrive at the so-called reduced wave equation. For instance, by applying the curl operator to (1) and substituting (2) into the result, we arrive at the magnetic field wave equation:

$$
\nabla \times(\tilde{\boldsymbol{\rho}} \nabla \times \mathbf{H})-i \omega \boldsymbol{\mu} \mathbf{H}=i \omega \boldsymbol{\mu} \mathbf{M}+\nabla \times(\tilde{\rho} \mathbf{J}),
$$

where $\tilde{\boldsymbol{\rho}}=\tilde{\boldsymbol{\sigma}}^{-1}$. The above equation is complemented with the condition that the electromagnetic fields are expected to decrease sufficiently fast when increasing the distance to the transmitter.

\subsection{Finite element formulation}

In this subsection, we derive the most common variational (weak) formulation used for finite element computations (see e.g., 4, 23]). Let $\mathbf{F}$ be an arbitrary test 
function and $\overline{\mathbf{F}}^{T}$ its conjugate transpose. Pre-multiplying Equation (4) by $\overline{\mathbf{F}}^{T}$ and integrating over domain $\Omega=\mathbb{R}^{3}$, we obtain:

$$
\begin{array}{r}
\int_{\Omega} \overline{\mathbf{F}}^{T}(\nabla \times \tilde{\boldsymbol{\rho}} \nabla \times \mathbf{H}) d \Omega-i \omega \mu_{0} \int_{\Omega} \overline{\mathbf{F}}^{T} \mathbf{H} d \Omega \\
=i \omega \mu_{0} \int_{\Omega} \overline{\mathbf{F}}^{T} \mathbf{M} d \Omega+\int_{\Omega} \overline{\mathbf{F}}^{T} \nabla \times(\tilde{\rho} \mathbf{J}) d \Omega
\end{array}
$$

By selecting $\mathbf{F}$ and $\mathbf{H}$ from the proper functional space that guarantees the integrability of the above formulation (see [23]), and using integration by parts, we obtain:

$$
b(\mathbf{F}, \mathbf{H})=l(\mathbf{F})
$$

where

$$
\begin{aligned}
b(\mathbf{F}, \mathbf{H})= & \int_{\Omega}(\nabla \times \overline{\mathbf{F}})^{T}(\tilde{\boldsymbol{\rho}} \nabla \times \mathbf{H}) d \Omega \\
& -i \omega \mu_{0} \int_{\Omega} \overline{\mathbf{F}}^{T} \mathbf{H} d \Omega, \\
l(\mathbf{F})= & i \omega \mu_{0} \int_{\Omega} \overline{\mathbf{F}}^{T} \mathbf{M} d \Omega+\int_{\Omega} \overline{\mathbf{F}}^{T} \nabla \times(\tilde{\rho} \mathbf{J}) d \Omega,
\end{aligned}
$$

\section{Derivative with respect to bed boundary position}

For the sake of simplicity on the derivation of the adjoint-formulation, in this Section we consider a media composed of only two layers, separated by a horizontal interface (see Figure 11). Nevertheless, the proposed method easily extends to an arbitrary number of interfaces. In Figure 1, $z_{i}$ indicates the vertical location of the planar interface that separates the two materials. We denote the corresponding material conductivity as $\boldsymbol{\sigma}_{z_{i}}$, which is given by:

$$
\boldsymbol{\sigma}_{z_{i}}=\left\{\begin{array}{l}
\boldsymbol{\sigma}^{-} z<z_{i} \\
\boldsymbol{\sigma}^{+} z>z_{i}
\end{array}\right.
$$

where $\boldsymbol{\sigma}^{+}$and $\boldsymbol{\sigma}^{-}$are strictly positive and constant TI tensors. In the following, subscript " $z_{i}$ " will indicate the material properties, electromagnetic fields, and variational formulations corresponding to this two-layer model problem. Thus, a subscript " $z_{i}+\epsilon$ " indicates the materials, fields, or variational formulations in the same model problem where now the interface is located at $z_{i}+\epsilon$.

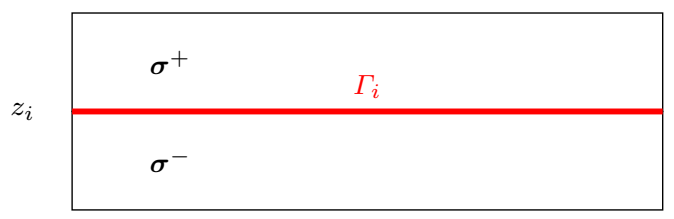

Fig. 1: 1D TI media composed of two different materials. 
We assume that the recorded geophysical resistivity measurements are given by:

$$
m\left(z_{i}\right)=\int_{\Omega} \overline{\mathbf{K}}^{T} \mathbf{H}_{z_{i}}+\int_{\Omega} \overline{\mathbf{G}}^{T}\left(\tilde{\rho}_{z_{i}} \nabla \times \mathbf{H}_{z_{i}}\right),
$$

for some $\mathbf{K}$ and $\mathbf{G}$. The first term in the right-hand-side of 9 corresponds to measurements of the magnetic field while the second one represents electric field measurements. In order to obtain the adjoint-based formula that expresses the derivative of the measurements, it is convenient to introduce the adjoint solution $\mathbf{H}_{z_{i}}^{*}$, which satisfies:

$$
b_{z_{i}}\left(\mathbf{H}_{z_{i}}^{*}, \mathbf{F}\right)=\int_{\Omega} \overline{\mathbf{K}}^{T} \mathbf{F} d \Omega+\int_{\Omega} \overline{\mathbf{G}}^{T}\left(\tilde{\rho}_{z_{i}} \nabla \times \mathbf{F}\right),
$$

where $\mathbf{H}_{z_{i}}^{*}$ is selected from the same space as $\mathbf{H}_{z_{i}}$. From a physical point of view, we obtain the adjoint solution by switching the roles that transmitters and receivers play in our problem. Therefore, we only change the right-hand-side of the original problem (6) to obtain the adjoint solution. As a result, we are able to reuse the matrix factorization used to solve (6), also for the adjoint problem (10). Thus, when using a direct solver, the additional cost of solving the adjoint problem is negligible in comparison to that of solving the original forward simulation (see e.g., [16]).

As a direct application of $(9)$ and $(10)$, we observe that we recover the measurement value by correlation of the direct and adjoint solutions:

$$
b_{z_{i}}\left(\mathbf{H}_{z_{i}}^{*}, \mathbf{H}_{z_{i}}\right)=m\left(z_{i}\right) \text {. }
$$

We emphasize that (10) and (11) are valid for any interface position $z_{i}$.

At that point, it is possible to differentiate (11) with respect to $z_{i}$ formally. As shown in [18, this technique is very efficient to compute the derivative of the measurement with respect to the conductivity values. Here, we are interested in computing the derivative with respect to the bed boundary position, and formally differentiate (11) leads to the introduction of complicated mathematical operators. To avoid this, in here, for a given position $z_{i}$, we introduce a small perturbation $\epsilon$ and recover the differential by letting $\epsilon \rightarrow 0$. We also mention that the interface must be infinitely long and that specific decaying conditions are assumed on the electromagnetic fields in the approach presented in [18. On the other hand, the approached proposed here applies in more general settings, including bounded domains.

Since the right-hand-side of 10 is independent of $\mathbf{H}_{z_{i}}^{*}$, if we select $\mathbf{F}:=\mathbf{H}_{z_{i}+\epsilon}$, we obtain the following identity:

$$
b_{z_{i}+\epsilon}\left(\mathbf{H}_{z_{i}+\epsilon}^{*}, \mathbf{H}_{z_{i}+\epsilon}\right)=b_{z_{i}}\left(\mathbf{H}_{z_{i}}^{*}, \mathbf{H}_{z_{i}+\epsilon}\right) .
$$

Similarly, by using (7), we have:

$$
b_{z_{i}}\left(\mathbf{H}_{z_{i}}^{*}, \mathbf{H}_{z_{i}}\right)=b_{z_{i}+\epsilon}\left(\mathbf{H}_{z_{i}}^{*}, \mathbf{H}_{z_{i}+\epsilon}\right) .
$$

By using Equation (11), and subtracting (13) from 12 , we obtain that the difference in the recorded measurements when the interface between the two layers 
is displaced by $\epsilon$ is given by the following integral:

$$
\begin{gathered}
m\left(z_{i}+\epsilon\right)-m\left(z_{i}\right)=b_{z_{i}}\left(\mathbf{H}_{z_{i}}^{*}, \mathbf{H}_{z_{i}+\epsilon}\right)-b_{z_{i}+\epsilon}\left(\mathbf{H}_{z_{i}}^{*}, \mathbf{H}_{z_{i}+\epsilon}\right) \\
=\int_{\Omega}\left(\nabla \times \overline{\mathbf{H}_{z_{i}}^{*}}\right)^{T}\left(\tilde{\boldsymbol{\rho}}_{z_{i}}-\tilde{\boldsymbol{\rho}}_{z_{i}+\epsilon}\right)\left(\nabla \times \mathbf{H}_{z_{i}+\epsilon}\right) d \Omega \\
=\int_{\mathbf{x}_{t}} \int_{z_{i}}^{z_{i}+\epsilon}\left(\nabla \times \overline{\mathbf{H}_{z_{i}}^{*}}\right)^{T}\left(\tilde{\boldsymbol{\rho}}_{z_{i}}-\tilde{\boldsymbol{\rho}}_{z_{i}+\epsilon}\right) \\
\cdot\left(\nabla \times \mathbf{H}_{z_{i}+\epsilon}\right) d x_{\mathbf{n}} d \mathbf{x}_{\mathbf{t}} .
\end{gathered}
$$

At this point, it is critical to treat separately the normal and tangential components of the curl since they satisfy different continuity conditions. The correct treatment of the continuity conditions is crucial to obtain the correct formula for the derivative. In particular, we obtain different weightings for the normal and tangential contributions of the electromagnetic fields at the interface. It turns out that it is easy to derive the correct formula in the setting considered in this work. On the other hand, it would be more challenging and mathematically technical to obtain the correct weightings when using a direct approach as in [18. For each component of the electric field, continuity of the electric displacement implies the following continuity conditions:

$$
\begin{aligned}
& {\left[\left(\mathbf{E}_{z_{i}+\epsilon}\right)_{\mathbf{t}}\right]_{z_{i}+\epsilon}=\mathbf{0},} \\
& {\left[\tilde{\sigma}_{\mathbf{n}, z_{i}+\epsilon}\left(\mathbf{E}_{z_{i}+\epsilon}\right)_{\mathbf{n}}\right]_{z_{i}+\epsilon}=0,}
\end{aligned}
$$

where, symbol [. $]_{z_{i}+\epsilon}$ denotes the jump across the interface, and $\tilde{\sigma}_{\mathbf{n}, z_{i}+\epsilon}=\sigma_{\mathbf{n}, z_{i}+\epsilon}-$ $i \omega \varepsilon_{0}$. By using (1), we obtain the following continuity conditions for the curl of the magnetic field:

$$
\begin{aligned}
& {\left[\tilde{\rho}_{\mathbf{t}, z_{i}+\epsilon}\left(\nabla \times \mathbf{H}_{z_{i}+\epsilon}\right)_{\mathbf{t}}\right] z_{i}+\epsilon=\mathbf{0},} \\
& {\left[\left(\nabla \times \mathbf{H}_{z_{i}+\epsilon}\right)_{\mathbf{n}}\right] z_{z_{i}+\epsilon}=0,}
\end{aligned}
$$

where $\tilde{\rho}_{\mathbf{t}, z_{i}+\epsilon}=\left(\tilde{\sigma}_{\mathbf{t}, z_{i}+\epsilon}\right)^{-1}$, and $\tilde{\sigma}_{\mathbf{t}, z_{i}+\epsilon}=\sigma_{\mathbf{t}, z_{i}+\epsilon}-i \omega \varepsilon_{0}$. Then, we separate explicitly the tangential and normal components in (14), and re-arrange them to obtain the following expression:

$$
\begin{aligned}
& m\left(z_{i}+\epsilon\right)-m\left(z_{i}\right) \\
& =\int_{\mathbf{x}_{\mathbf{t}}} \int_{z_{i}}^{z_{i}+\epsilon}\left(\nabla \times \overline{\mathbf{H}_{z_{i}}^{*}}\right)_{\mathbf{t}}^{T}\left(\tilde{\rho}_{\mathbf{t}, z_{i}}-\tilde{\rho}_{\mathbf{t}, z_{i}+\epsilon}\right) \\
& +\left(\nabla \times \mathbf{H}_{z_{i}+\epsilon}\right)_{\mathbf{t}} d x_{\mathbf{n}} d \mathbf{x}_{\mathbf{t}} \\
& +\int_{\mathbf{x}_{\mathbf{t}}} \int_{z_{i}}^{z_{i}+\epsilon}\left(\nabla \times \overline{\mathbf{H}_{z_{i}}^{*}}\right)_{\mathbf{n}}^{T}\left(\tilde{\rho}_{\mathbf{n}, z_{i}}-\tilde{\rho}_{\mathbf{n}, z_{i}+\epsilon}\right) \\
& =-\int_{\mathbf{x}_{\mathbf{t}}} \int_{z_{i}}^{z_{i}+\epsilon}\left(\tilde{\sigma}_{\mathbf{t}, z_{i}}-\tilde{\sigma}_{\mathbf{t}, z_{i}+\epsilon}\right)\left(\tilde{\rho}_{\mathbf{t}, z_{i}} \nabla \times \overline{\mathbf{H}_{z_{i}}+\epsilon}\right)_{\mathbf{t}}^{T} d x_{\mathbf{n}} d \mathbf{x}_{\mathbf{t}} \\
& \cdot\left(\tilde{\rho}_{\mathbf{t}, z_{i}+\epsilon} \nabla \times \mathbf{H}_{z_{i}+\epsilon}\right)_{\mathbf{t}} d x_{\mathbf{n}} d \mathbf{x}_{\mathbf{t}} \\
& +\int_{\mathbf{x}_{\mathbf{t}}} \int_{z_{i}}^{z_{i}+\epsilon}\left(\nabla \times \overline{\mathbf{H}_{z_{i}}^{*}}\right)_{\mathbf{n}}^{T}\left(\tilde{\rho}_{\mathbf{n}, z_{i}}-\tilde{\rho}_{\mathbf{n}, z_{i}+\epsilon}\right) \\
& \cdot\left(\nabla \times \mathbf{H}_{z_{i}+\epsilon}\right)_{\mathbf{n}} d x_{\mathbf{n}} d \mathbf{x}_{\mathbf{t}},
\end{aligned}
$$


where $\tilde{\rho}_{\mathbf{n}, z_{i}}=\left(\tilde{\sigma}_{\mathbf{n}, z_{i}}\right)^{-1}$. Recalling (16), we have:

$$
\begin{aligned}
\tilde{\rho}_{\mathbf{t}, z_{i}+\epsilon}\left(\nabla \times \mathbf{H}_{z_{i}+\epsilon}\right)_{\mathbf{t}} & =\tilde{\rho}_{\mathbf{t}, z_{i}}\left(\nabla \times \mathbf{H}_{z_{i}}\right)_{\mathbf{t}}+o(\epsilon), \\
\left(\nabla \times \mathbf{H}_{z_{i}+\epsilon}\right)_{\mathbf{n}} & =\left(\nabla \times \mathbf{H}_{z_{i}}\right)_{\mathbf{n}}+o(\epsilon) .
\end{aligned}
$$

We point out that 18 implies that the tangential and normal components of the curl exhibit different convergence behaviors as $\epsilon \rightarrow 0$. These different behaviors are complicated to understand when differentiating (11) directly, and are of paramount importance to derive the correct formula.

Using Taylor's series expansion, we show that for any smooth function $\psi$, we have:

$$
\begin{aligned}
& \int_{\Omega}\left(\tilde{\rho}_{\mathbf{n}, z_{i}}-\tilde{\rho}_{\mathbf{n}, z_{i}+\epsilon}\right) \psi\left(\mathbf{x}_{\mathbf{t}}, z\right) d \mathbf{x} \\
&= \int_{\mathbf{x}_{\mathbf{t}}} \int_{z_{i}}^{z_{i}+\epsilon}\left(\tilde{\rho}_{\mathbf{n}, z_{i}}-\tilde{\rho}_{\mathbf{n}, z_{i}+\epsilon}\right) \psi\left(\mathbf{x}_{\mathbf{t}}, z\right) d x_{\mathbf{n}} d \mathbf{x}_{\mathbf{t}} \\
&=-\int_{\mathbf{x}_{\mathbf{t}}} \int_{z_{i}}^{z_{i}+\epsilon}\left[\tilde{\rho}_{\mathbf{n}}\right]\left(\psi\left(\mathbf{x}_{\mathbf{t}}, z_{i}\right)+o(\epsilon)\right) d x_{\mathbf{n}} d \mathbf{x}_{\mathbf{t}} \\
&=-\int_{\mathbf{x}_{\mathbf{t}}} \epsilon\left[\tilde{\rho}_{\mathbf{n}}\right]\left(\psi\left(\mathbf{x}_{\mathbf{t}}, z_{i}\right)+o(\epsilon)\right) d \mathbf{x}_{\mathbf{t}} \\
&=-\epsilon\left[\tilde{\rho}_{\mathbf{n}}\right] \int_{\Gamma_{z_{i}}} \psi\left(\mathbf{x}_{\mathbf{t}}, z_{i}\right) d \Gamma_{z_{i}}+o\left(\epsilon^{2}\right),
\end{aligned}
$$

where $\Gamma_{z_{i}}$ is the interface between the two materials. Following an analogous argument for the tangential component, and summing up both tangential and normal components according to (17), we obtain:

$$
\begin{aligned}
& \frac{m\left(z_{i}+\epsilon\right)-m\left(z_{i}\right)}{\epsilon} \\
& =\left[\tilde{\sigma}_{\mathbf{t}}\right] \int_{\Gamma_{z_{i}}}\left(\tilde{\rho}_{\mathbf{t}, z_{i}}\left(\nabla \times \overline{\mathbf{H}_{z_{i}}^{*}}\right)_{\mathbf{t}}\right)^{T} \\
& \quad \cdot\left(\tilde{\rho}_{\mathbf{t}, z_{i}}\left(\nabla \times \mathbf{H}_{z_{i}}\right)_{\mathbf{t}}\right) d \Gamma_{z_{i}} \\
& \quad-\left[\tilde{\rho}_{\mathbf{n}}\right] \int_{\Gamma_{z_{i}}}\left(\nabla \times \overline{\mathbf{H}_{z_{i}}^{*}}\right)_{\mathbf{n}}^{T}\left(\nabla \times \mathbf{H}_{z_{i}}\right)_{\mathbf{n}} d \Gamma_{z_{i}}+o(\epsilon) .
\end{aligned}
$$

Finally, letting $\epsilon \rightarrow 0$, we have:

$$
\begin{aligned}
\frac{d m}{d z_{i}}\left(z_{i}\right) & =\left[\tilde{\sigma}_{\mathbf{t}}\right] \int_{\Gamma_{z_{i}}}\left(\tilde{\rho}_{\mathbf{t}, z_{i}} \nabla \times \overline{\mathbf{H}_{z_{i}}^{*}}\right)_{\mathbf{t}}^{T}\left(\tilde{\rho}_{\mathbf{t}, z_{i}} \nabla \times \mathbf{H}_{z_{i}}\right)_{\mathbf{t}} d \Gamma_{z_{i}} \\
& -\left[\tilde{\rho}_{\mathbf{n}}\right] \int_{\Gamma_{z_{i}}}\left(\nabla \times \overline{\mathbf{H}_{z_{i}}^{*}}\right)_{\mathbf{n}}^{T}\left(\nabla \times \mathbf{H}_{z_{i}}\right)_{\mathbf{n}} d \Gamma_{z_{i}} .
\end{aligned}
$$

From the geophysical point of view, Equation (21) implies that we need to treat differently the normal and tangential components of the electromagnetic fields in order to find the derivatives of the acquired measurements with respect to the bed boundary positions. This separate treatment is due to the different continuity conditions that exist for the different components of the electromagnetic fields. 


\section{Numerical experiments}

4.1 Model problem A: 2D potential equation

In this subsection, we verify our proposed adjoint-based method to compute derivatives with respect to bed boundary positions, and we analyze their convergence speed with respect to the finite element mesh size. To do so, we consider a simple scenario in which $\omega=0$. We further assume a 2D problem in the transverse magnetic polarization, so that $H$ is a scalar and $\mathbf{E}$ is vector with two components. Then (2) shows that $\nabla \times \mathbf{E}=0$, so that $\mathbf{E}=\nabla \phi$ for some scalar potential $\phi$. Such scalar potential satisfies the continuity equation $\nabla \cdot(\sigma \nabla \phi)=\nabla \cdot \mathbf{J}$, which governs the electrostatic phenomena in a lossy media (see, e.g., [24]). Furthermore, we can use (21) with $\nabla \times H=\sigma \nabla \phi+\mathbf{J}$.

To better analyze the numerical results, we consider a simple problem for which we know the exact solution. Thus, we select $\mathbf{M}=\mathbf{0}$ and $\mathbf{J}=\left(0,2 \mathbf{x}_{2}\right)$, so that $\nabla \cdot \mathbf{J}=2$. The domain is the unit square $\Omega=(0,1)^{2}$. Thus, $\phi_{z_{i}}$ is solution to

$$
-\nabla \cdot\left(\sigma_{z_{i}} \nabla \phi_{z_{i}}\right)=-2
$$

where $\boldsymbol{\sigma}_{z_{i}}=\sigma_{z_{i}} \mathbf{I}_{2}$, with

$$
\sigma_{z_{i}}(\mathbf{x})= \begin{cases}\sigma_{-} & \text {if } z<z_{i} \\ \sigma_{+} & \text {if } z>z_{i}\end{cases}
$$

is the conductivity of a $1 \mathrm{D}$ layered media that consists of two different layers, and $\sigma_{ \pm}$are two positive values. In this example, we consider $\sigma_{-}=1 \mathrm{Sm}^{-1}$ and $\sigma_{+}=10 S m^{-1}$, and $f=-2$. The boundary conditions are:

$$
\begin{gathered}
\phi_{z_{i}}(x, 0)=\frac{\partial \phi_{z_{i}}}{\partial z}(x, 1) \\
=0, \\
\frac{\partial \phi_{z_{i}}}{\partial x}(0, z)=\frac{\partial \phi_{z_{i}}}{\partial x}(1, z) \\
=0 .
\end{gathered}
$$

The analytical solution for this problem is:

$$
\phi_{z_{i}}(\mathbf{x})= \begin{cases}\rho_{-} z(z-2) & \text { if } z<z_{i} \\ \rho_{+} z(z-2)-[\rho] z_{i}\left(z_{i}-2\right) & \text { if } z>z_{i}\end{cases}
$$

For the measurements, we consider $\mathbf{K}=\mathbf{M}=\mathbf{0}$ and $\mathbf{G}=\mathbf{J}=\left(0,2 \mathbf{x}_{2}\right)$ so that the direct and adjoint solutions are the same. Measurements are then given by:

$$
\begin{aligned}
& m\left(z_{i}\right)=\int_{\Omega} \nabla \times(\tilde{\rho} \mathbf{J}) H_{z_{i}} d \Omega=\int_{\Omega} \mathbf{J} \tilde{\rho} \nabla \times\left(H_{z_{i}}\right) d \Omega \\
& =\int_{\Omega} \mathbf{J} \nabla \phi_{z_{i}}+\int_{\Omega}|\mathbf{J}|^{2} d \Omega=-\int_{\Omega} \nabla \cdot \mathbf{J} \phi_{z_{i}} d \Omega+\int_{\Omega}|\mathbf{J}|^{2} d \Omega \\
& =-2 \int_{\Omega} \phi_{z_{i}} d \Omega+\frac{4}{3}=-2[\rho]\left(\frac{2}{3} z_{i}^{3}-2 z_{i}^{2}+2 z_{i}\right)+\frac{4}{3} \rho_{+}+\frac{4}{3} .
\end{aligned}
$$

Thus:

$$
\frac{d m}{d z_{i}}\left(z_{i}\right)=-4[\rho]\left(z_{i}-1\right)^{2}
$$


Figure 2 compares the derivative of $m$ computed using adjoint state expression (21) vs. analytical expression (26). As shown there, the numerical result is highly accurate. Figure 3 analyses the convergence of the adjoint-based gradient with respect to the finite element mesh size for the case, $z_{i}=0.5$, and we observe a linear convergence rate.

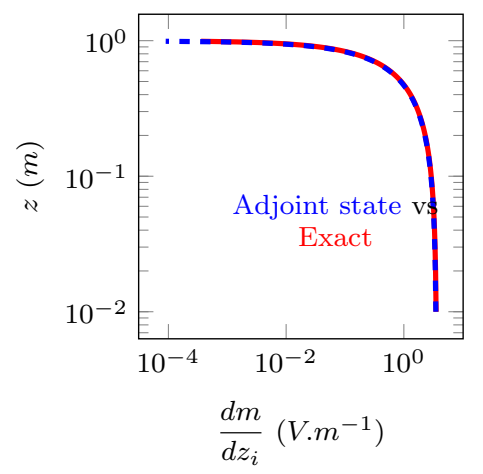

Fig. 2: Model problem A. Analytical and numerical gradient.

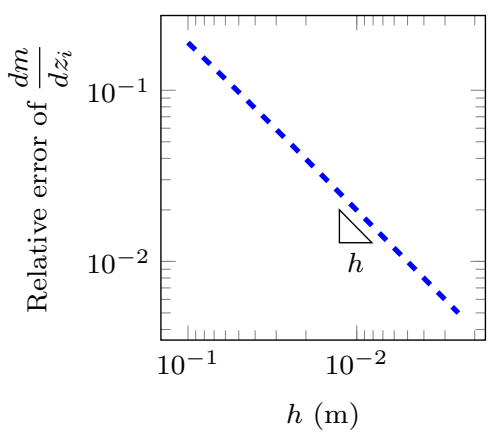

Fig. 3: Model problem A. Convergence of the numerical gradient at point $z_{i}=0.5$.

\subsection{5D borehole application}

In this model problem, we consider a 1D layered formation. Moreover, we assume to have a co-axial tool in a vertical well. Thus, the source is given by a Vertical Magnetic Dipole (VMD). We solve the aforementioned model problem by using a $1.5 \mathrm{D}$ variational formulation described in [12] (see Equation 48) of Appendix B). 


\subsubsection{Model problem B: two-layer media}

Figure 4 describes the logging instrument used in this model problem. The conductivity of the two-layer media is given by $\boldsymbol{\sigma}_{z_{i}}=\sigma_{z_{i}} \mathbf{I}_{3}$, where:

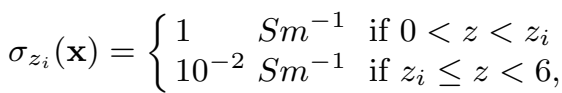

and $z_{i}=3.15 \mathrm{~m}$.

In this example, the measurement is the value of the $z$ component of the magnetic field at the receiver. To simplify notation, we denote $H=H_{z_{i}, z}(R x)$ to the recorded value, and we have $m\left(z_{i}\right)=H$.

Figure 5 shows the real and imaginary parts and the absolute value of $H$ for different tool positions. Figure 6 compares the derivative with respect to the bed boundary position using the 1.5D adjoint formulation (48) vs. that obtained with a finite differences approximation. The finite differences approximation experiences some oscillations due to numerical errors. The solution using the adjoint state method shows superior accuracy and avoids any spurious oscillation.

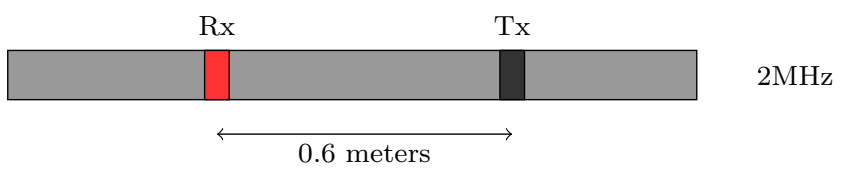

Fig. 4: Logging instrument of model problem B. Logging instrument. Tx and Rx denote the transmitter and the receiver, respectively.

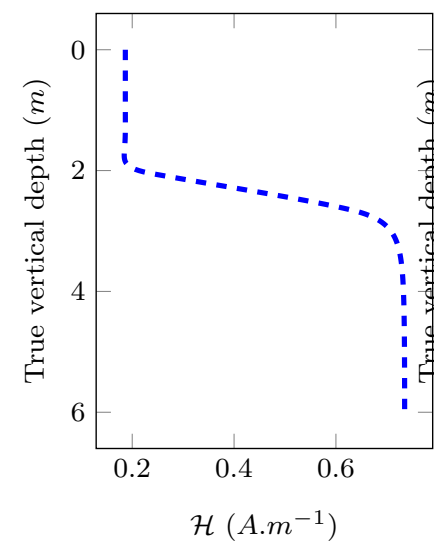

(a) Real part

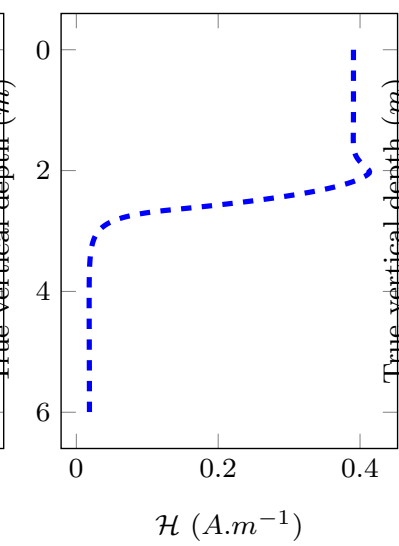

(b) Imaginary part

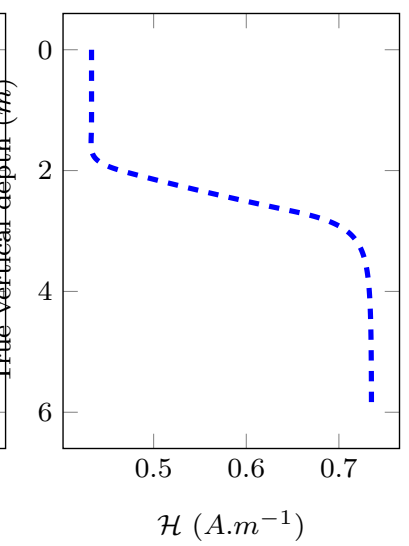

(c) Absolute value

Fig. 5: Model problem B. $z z$-component of the magnetic field for a vertical well. 

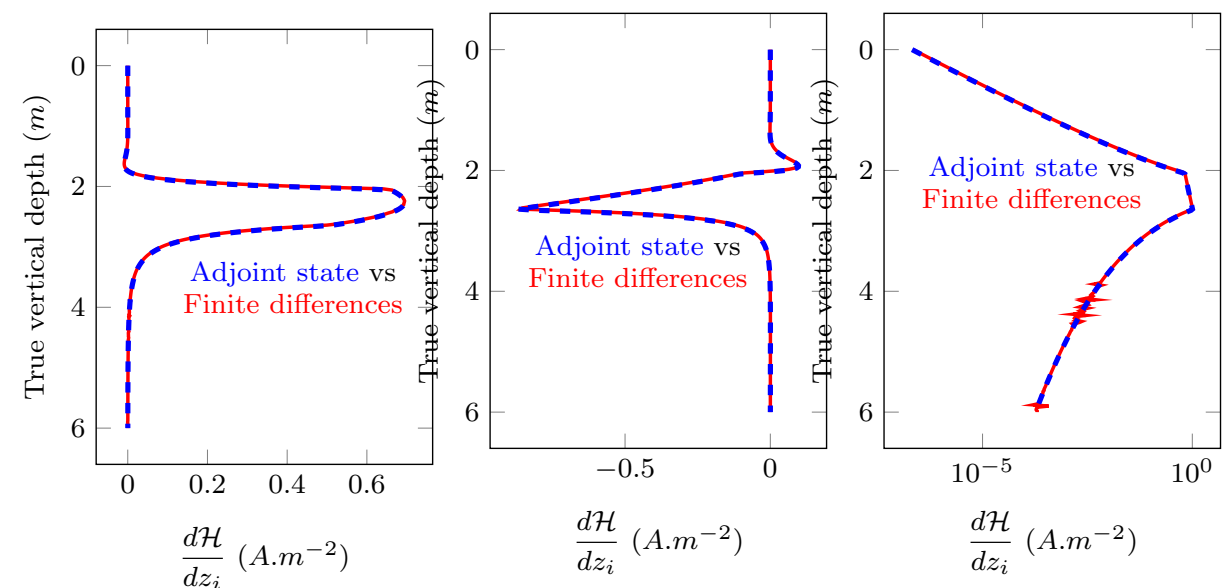
(a) Real part of the derivative $\begin{aligned} & \text { (b) Imaginary part of the (c) Absolute value of the } \\ & \text { derivative }\end{aligned}$

Fig. 6: Model problem B. Derivative with respect to bed boundary position of the $z z$-component of the magnetic field for a vertical well.

\subsubsection{Model problem C: multi-layer media}

Figure 7 describes the logging instrument used for this model problem. In this example, the conductivity model features four layers. $\mathbf{z}_{i}=\left(z_{i, 1}, z_{i, 2}, z_{i, 3}\right)$ represents the location of the three interfaces. The conductivity of the media is $\boldsymbol{\sigma}_{\mathbf{z}_{i}}=\sigma_{\mathbf{z}_{i}} \mathbf{I}_{3}$, where:

$$
\sigma_{\mathbf{z}_{i}}(\mathbf{x})=\left\{\begin{array}{lll}
1 & S m^{-1} & \text { if } 0<z<z_{i, 1} \\
10^{-2} & S m^{-1} & \text { if } z_{i, 1} \leq z<z_{i, 2} \\
1 & S m^{-1} & \text { if } z_{i, 2} \leq z<z_{i, 3} \\
10^{-2} & S m^{-1} & \text { if } z_{i, 3} \leq z<10
\end{array}\right.
$$

with $\mathbf{z}_{i}=(3,5,7)$. For this problem, we consider the attenuation and the phase

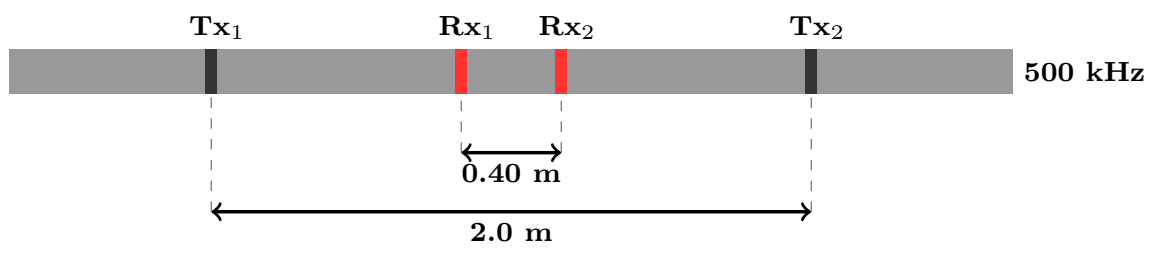

Fig. 7: Logging instrument of model problem $\mathrm{C} . \mathrm{Rx}_{1}$ and $\mathrm{Rx}_{2}$ are the first and the second receivers, respectively. $\mathrm{Tx}_{1}$ and $\mathrm{Tx}_{2}$ are the first and the second transmitters, respectively.

difference of the magnetic field between the two receivers. These are the quantities often recorded in borehole geophysical measurements. For details, see Appendix A 
Figure 8 describes the attenuation and the phase difference for the $z z$-component of the magnetic field. Figures 9 and 10 show the derivatives of the attenuation and the phase difference with respect to all bed boundary positions of the media using the adjoint state formulation vs. those obtained with a finite difference method. As shown in the figures, the derivatives using the adjoint state method coincide with the finite differences ones for all cases. Indeed, the adjoint-based derivatives produce enhanced accuracy (see Figure 11 displaying a zoom of the derivative). Additionally, the adjoint-based method only requires the solution of one finite element problem with two right hand sides, while the finite differences approach involves the solution of one additional problem per interface (i.e., a total of $N_{\text {int }}+1$ problems, where $N_{\text {int }}$ is the number of interfaces whose derivative is estimated).

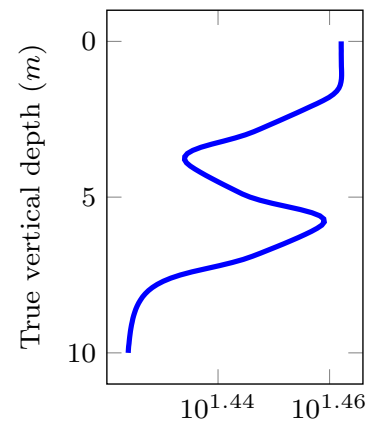

Attenuation $\mathcal{A}(\mathrm{dB})$

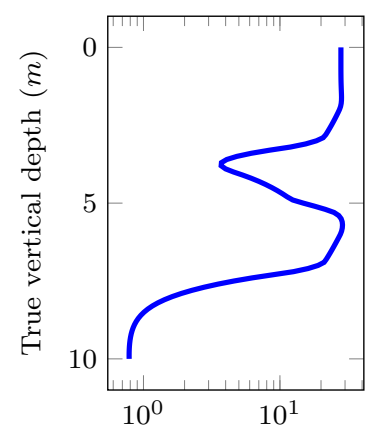

Phase difference $\mathcal{P}$ (degree)

Fig. 8: Model problem C. Attenuation and phase difference for the $z z$-component of the magnetic field.

\subsection{Model problem D: sequence of 1D media.}

In this model problem, we consider the short spacing deep azimuthal logging instrument shown in Figure 12. In recent years, deep azimuthal logging instruments were introduced to better navigate the borehole inside the reservoir, and avoid penetrating a water-saturated rock. Figure 13 shows our model formation, which consist of three 1D media. Figure 14 describes the simulated attenuations and phase differences of the $z z$ coupling of the magnetic field. Figure 15 exhibits the derivative of the attenuations with respect to both boundary positions crossed by the well trajectory. As evident in the figure, the sensitivity of the attenuation to a bed boundary increases while we are approaching it. Analogously, Figure 16 shows the derivatives of the phase differences with respect to the bed boundary positions.

\section{Conclusions}

We have developed an adjoint-based formulation to compute the derivatives of geophysical resistivity measurements with respect to the bed boundary positions. We 


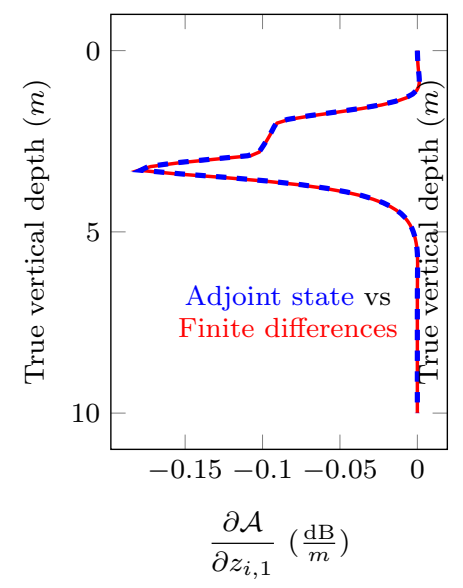

(a) First interface

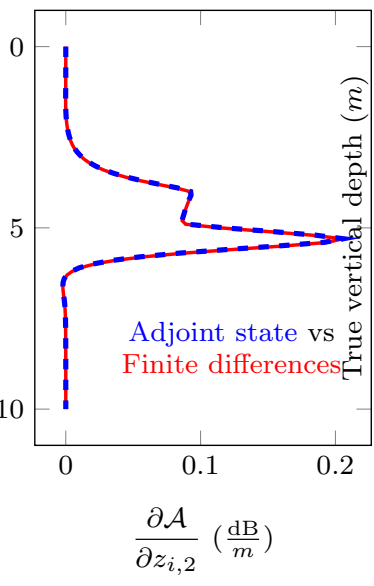

(b) Second interface

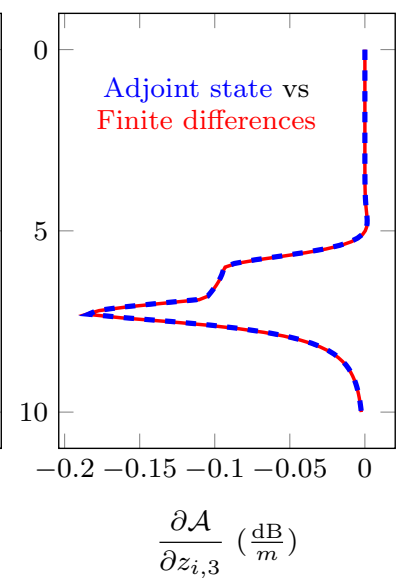

(c) Third interface

Fig. 9: Model problem C. Derivatives of the attenuation with respect to the bed boundary positions.

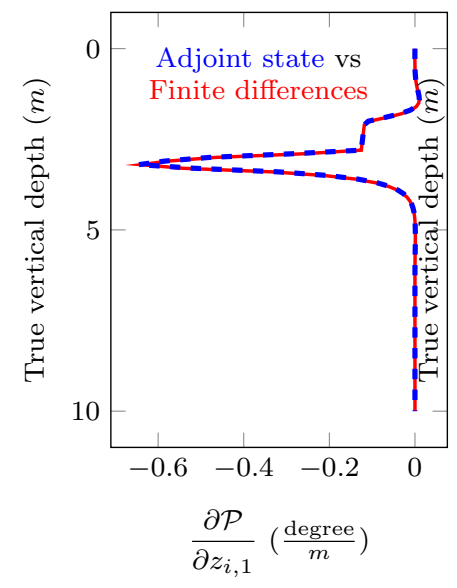

(a) First interface

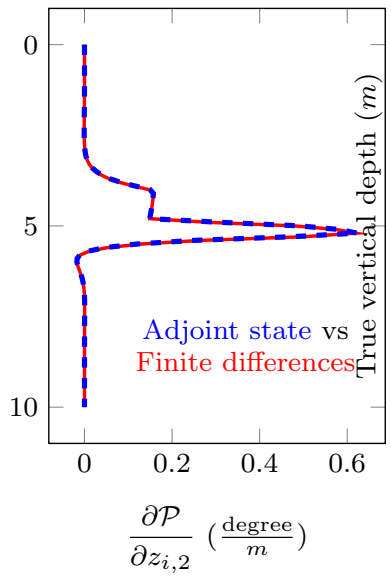

(b) Second interface

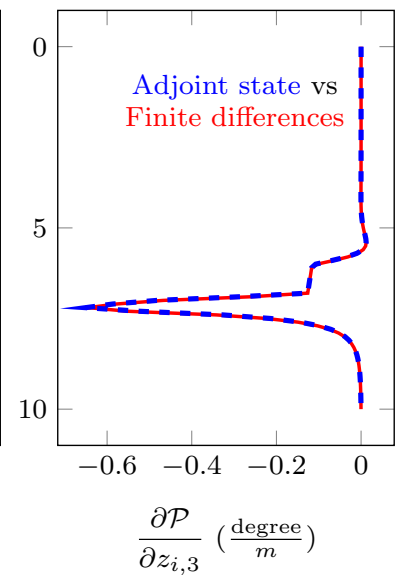

(c) Third interface

Fig. 10: Model problem C. Derivatives of the phase difference with respect to the bed boundary positions.

verified our formulations by comparing the numerical results with those obtained using an analytical solution for a potential equation and with a finite differences technique for a 1.5D Maxwell's system. Using the adjoint state method, we can compute the derivatives at (almost) no additional cost in time with respect to that needed to solve the forward problem, and we obtain an accurate evaluation of the derivatives. The formulations are valid for triaxial tools with arbitrary trajectories. 
Adjoint state vs

Finite differences

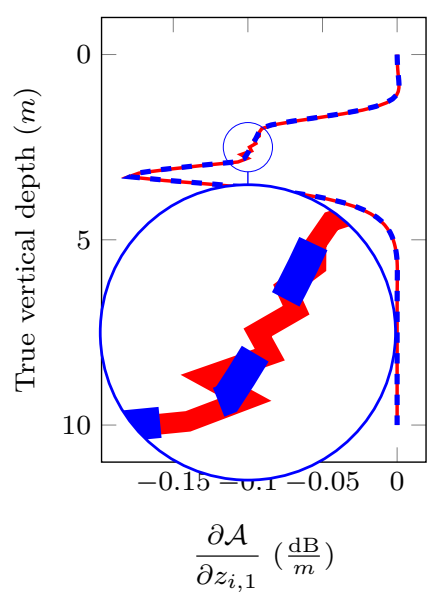

Fig. 11: Model problem C. Derivative of the attenuation with respect to the first bed boundary position.

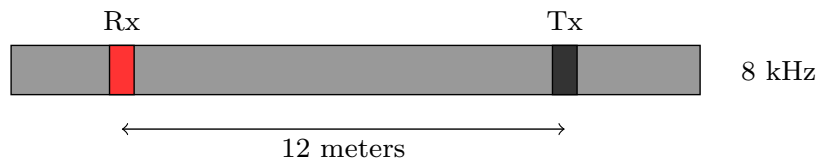

Fig. 12: Model problem D. Short spacing deep azimuthal instrument.

\section{Acknowledgement}

This work has received funding from the European Union's Horizon 2020 research and innovation programme under the Marie Sklodowska-Curie grant agreement No 777778, the Projects of the Spanish Ministry of Economy and Competitiveness with reference MTM2016-76329-R (AEI/FEDER, EU), and MTM2016-81697$\mathrm{ERC}$

-/AEI, the BCAM "Severo Ochoa" accreditation of excellence SEV-2013-0323, and the Basque Government through the BERC 2014-2017 program, and the Consolidated Research Group Grant IT649-13 on "Mathematical Modeling, Simulation, and Industrial Applications (M2SI)".

\section{A Derivatives of the attenuation and the phase difference}

In practice, LWD tools often measure the so-called attenuation and phase difference because of their relative insensitivity to the borehole size and mud resistivity. We post-process the measured aforementioned values to obtain two apparent resistivity values using a table look-up algorithm based on the measured values of homogeneous isotropic media with known resistivities (see, e.g., 25, 26]). 


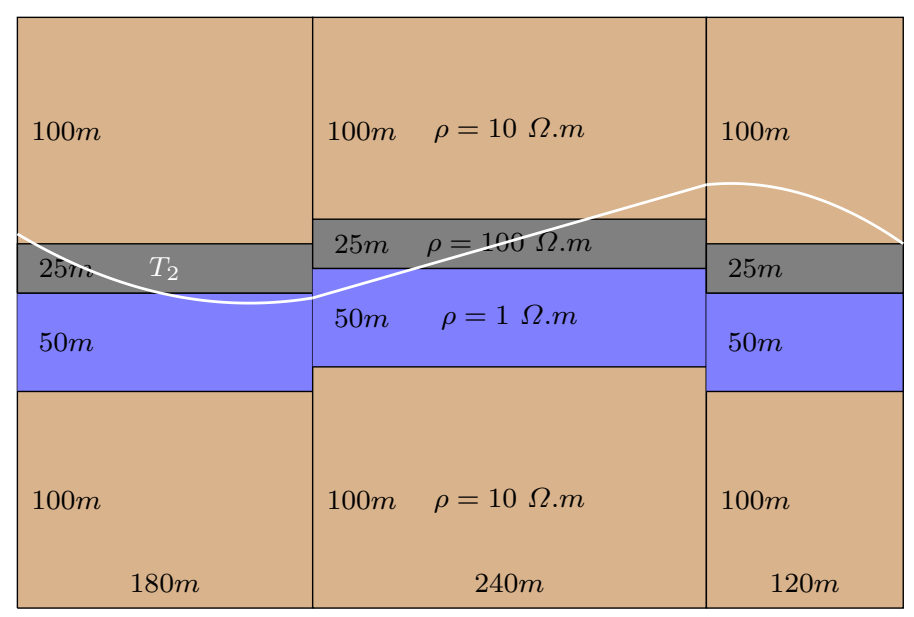

Fig. 13: Model problem D. A sequence of $1 \mathrm{D}$ models. The white line exhibits the trajectory.

This Appendix depicts how to compute the derivatives of the attenuation and the phase difference with respect to the bed boundary positions. To simplify, we introduce the notation

$$
H_{l}^{k}=H_{z_{i}, z}^{k}\left(R x_{l}\right), \quad(1 \leq k, l \leq 2)
$$

to denote the quantity measured at the receiver $l$ when the transmitter $k$ is active. For $l=1,2$, attenuation $A^{l}$ and phase difference $P^{l}$ are defined from $H_{1}^{l}$ and $H_{2}^{l}$ as:

$$
\ln \frac{H_{1}^{l}}{H_{2}^{l}}=\underbrace{\ln \frac{\left|H_{1}^{l}\right|}{\left|H_{2}^{l}\right|}}_{\times 20 \log (e)=A^{l}(\mathrm{~dB})}+i \underbrace{\left(p h\left(H_{1}^{l}\right)-p h\left(H_{2}^{l}\right)\right)}_{\times \frac{180}{\pi}=P^{l}(\text { degree })},
$$

where $p h$ denotes the phase of a complex number. The final attenuation $A$ and phase difference $P$ are defined by averaging:

$$
A=\frac{1}{2}\left(A^{1}+A^{2}\right), \quad P=\frac{1}{2}\left(P^{1}+P^{2}\right) .
$$

When the instruments contains a single receiver, we have an analogous definition to 30 with $H_{2}^{l}=1$.

It remains to compute the derivative of $A^{l}$ and $P^{l}$ with respect to the position $z_{i, m}$ of the $m^{t h}$ bed boundary. We have

$$
\begin{aligned}
A^{l} & :=\ln \frac{\left|H_{1}^{l}\right|}{\left|H_{2}^{l}\right|}=\ln \frac{\sqrt{\left(H_{1}^{l, r e}\right)^{2}+\left(H_{2}^{l, i m}\right)^{2}}}{\sqrt{\left(H_{2}^{l, r e}\right)^{2}+\left(H_{2}^{l, i m}\right)^{2}}} \\
& =\frac{1}{2} \ln \left[\left(H_{1}^{l, r e}\right)^{2}+\left(H_{1}^{l, i m}\right)^{2}\right] \\
& -\frac{1}{2} \ln \left[\left(H_{2}^{l, r e}\right)^{2}+\left(H_{2}^{l, i m}\right)^{2}\right]
\end{aligned}
$$




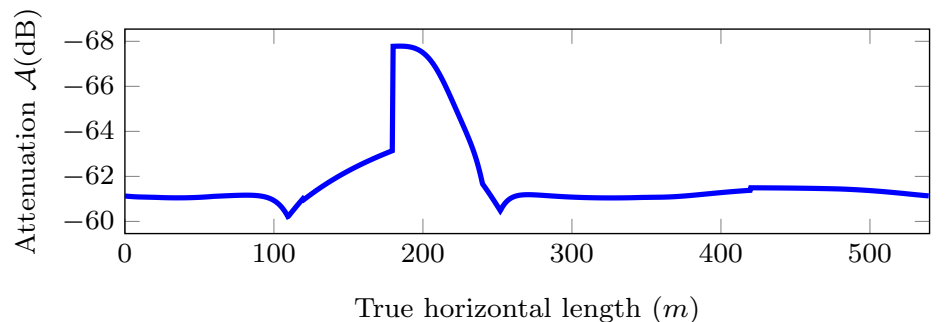

(a) Attenuations

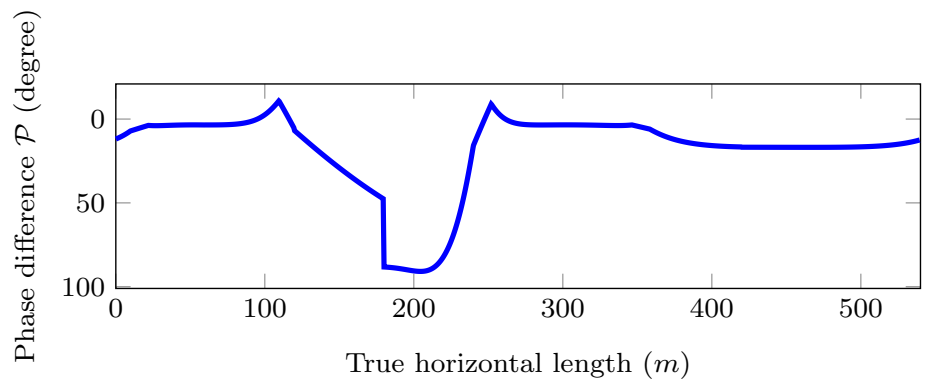

(b) Phase differences

Fig. 14: Model problem D. Attenuations and phase differences of the $z z$ coupling of the magnetic field.

where $H_{1}=H_{1}^{l, r e}+i H_{1}^{l, i m}$ and $H_{2}=H_{2}^{l, r e}+i H_{2}^{l, i m}$. Using the chain rule, we obtain:

$$
\begin{aligned}
\frac{\partial A}{\partial z_{i, m}} & =\frac{1}{\left|H_{1}^{l}\right|^{2}}\left(H_{1}^{l, r e} \frac{\partial H_{1}^{l, r e}}{\partial z_{i, m}}+H_{1}^{l, i m} \frac{\partial H_{1}^{l, i m}}{\partial z_{i, m}}\right) \\
& -\frac{1}{\left|H_{2}^{l}\right|^{2}}\left(H_{2}^{l, r e} \frac{\partial H_{2}^{l, r e}}{\partial z_{i, m}}+H_{2}^{l, i m} \frac{\partial H_{2}^{l, i m}}{\partial z_{i, m}}\right)
\end{aligned}
$$

For the phase difference, it holds that

$$
\begin{aligned}
P\left(\mathbf{z}_{i}\right) & :=p h\left(H_{1}\right)-p h\left(H_{2}\right) \\
& =\arctan \left(\frac{H_{1}^{l, i m}}{H_{1}^{l, r e}}\right)-\arctan \left(\frac{H_{2}^{l, i m}}{H_{2}^{l, r e}}\right) .
\end{aligned}
$$

Using again the chain rule, we obtain:

$$
\begin{aligned}
\frac{\partial P}{\partial z_{i, m}} & =\frac{1}{\left|H_{1}^{l}\right|^{2}}\left(H_{1}^{l, r e} \frac{\partial H_{1}^{l, i m}}{\partial z_{i, m}}-H_{1}^{l, i m} \frac{\partial H_{1}^{l, r e}}{\partial z_{i, m}}\right) \\
& -\frac{1}{\left|H_{2}^{l}\right|^{2}}\left(H_{2}^{l, r e} \frac{\partial H_{2}^{l, i m}}{\partial z_{i, m}}-H_{2}^{l, i m} \frac{\partial H_{2}^{l, r e}}{\partial z_{i, m}}\right) .
\end{aligned}
$$

\section{B 1.5D formulation}




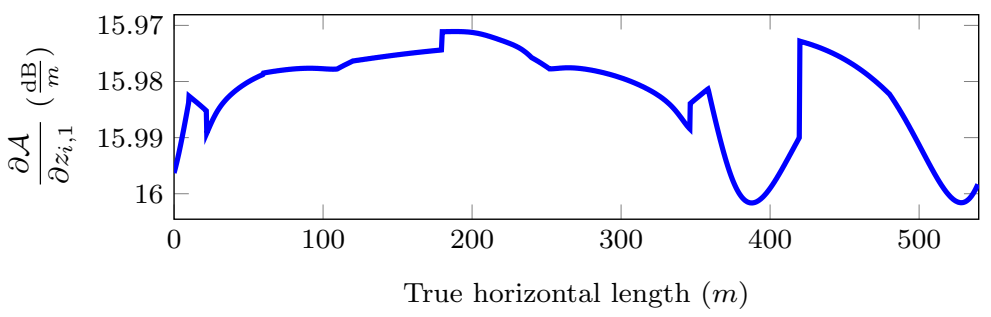

(a) First interface

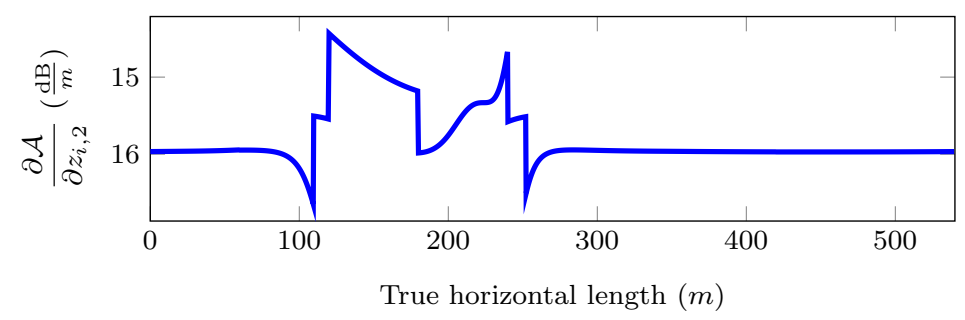

(b) Second interface

Fig. 15: Model problem D. Derivative of the attenuations with respect to bed boundary positions crossed by the well trajectory.

In this appendix, we follow 12 to derive the finite element (variational) formulation of a 1.5D resistivity problem. Then, we show how to compute the derivative with respect to the bed boundary positions according to Equation 21).

We consider the magnetic field in the Cartesian system of coordinates as $\mathbf{H}=\left(H_{x}, H_{y}, H_{z}\right)$. For problems where material properties vary only in the $z$-direction, it is convenient to use a 2D Fourier transform along the $x y$-plane. We consider $\widehat{\mathbf{H}}$ to be the 2D Fourier transform of $\mathbf{H}$ along $x$ and $y$ directions. We have:

$$
\mathbf{H}\left(\mathbf{x}_{t}, x_{n}\right):=\frac{1}{4 \pi^{2}} \int_{-\infty}^{+\infty} \int_{-\infty}^{+\infty} \widehat{\mathbf{H}}\left(\mathbf{k}, x_{n}\right) e^{i \mathbf{k} \cdot \mathbf{x}_{t}} d \mathbf{k}
$$

where $\mathbf{k}=\left(k_{x}, k_{y}\right)$. We change the system of coordinates from the Cartesian to a cylindrical one according to the following transformations:

$$
\begin{aligned}
& x=\rho \cdot \cos \phi, y=\rho \cdot \sin \phi, \\
& k_{x}=\xi \cdot \cos \theta, k_{y}=\xi \cdot \sin \theta .
\end{aligned}
$$

Substituting 37 into 36 , we obtain:

$$
\begin{aligned}
\mathbf{H}(\boldsymbol{\rho})=\frac{1}{4 \pi^{2}} \int_{0}^{+\infty} \int_{0}^{2 \pi} & \widehat{\mathbf{H}}\left(\xi, \theta, x_{n}\right) \\
& \cdot e^{i \xi \rho(\cos \theta \cos \phi+\sin \theta \sin \phi)} d \theta \xi d \xi,
\end{aligned}
$$

where $\rho=\left(\rho, \phi, x_{n}\right)$. Using the identity $\cos (\phi-\theta)=\cos \theta \cos \phi+\sin \theta \sin \phi$, we arrive at:

$$
\mathbf{H}(\boldsymbol{\rho})=\frac{1}{4 \pi^{2}} \int_{0}^{+\infty} \int_{0}^{2 \pi} \widehat{\mathbf{H}}\left(\xi, \theta, x_{n}\right) e^{i \xi \rho \cos (\phi-\theta)} d \theta \xi d \xi .
$$

We have the following relation between exponentials and Bessel functions:

$$
e^{i \xi \rho \cos (\phi-\theta)}=\sum_{r=-\infty}^{\infty} i^{r} J_{r}(\xi \rho) e^{-i r(\phi-\theta)} .
$$




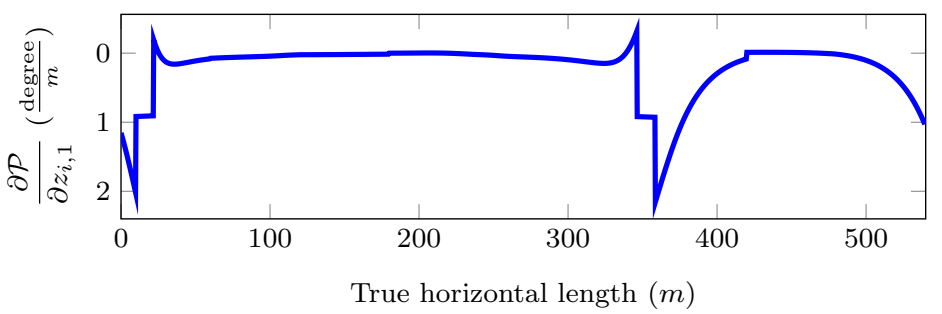

(a) First interface

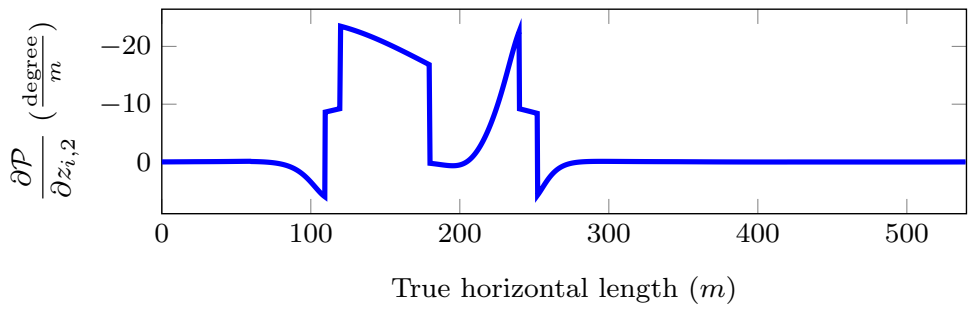

(b) Second interface

Fig. 16: Model problem D. Derivative of the phase differences with respect to bed boundary positions crossed by the well trajectory.

Substituting 40 into 39 , we obtain:

$$
\mathbf{H}(\boldsymbol{\rho})=\frac{1}{2 \pi} \sum_{r=-\infty}^{\infty} \int_{0}^{+\infty} \mathbf{H}^{r}\left(\xi, x_{n}\right) J_{r}(\xi \rho) e^{-i r \phi} \xi d \xi
$$

where

$$
\mathbf{H}^{r}\left(\xi, x_{n}\right)=\frac{1}{2 \pi} \int_{0}^{2 \pi} \widehat{\mathbf{H}}\left(\xi, \theta, x_{n}\right) i^{r} e^{i r \theta} d \theta .
$$

Analogously, we consider the adjoint solution as follows:

$$
\mathbf{H}^{*}(\boldsymbol{\rho})=\frac{1}{2 \pi} \sum_{r=-\infty}^{\infty} \int_{0}^{+\infty} \mathbf{H}^{*, r}\left(\xi, x_{n}\right) J_{r}(\xi \rho) e^{-i r \phi} \xi d \xi
$$

For an arbitrary function $\mathbf{g}(\xi, z)=\left(g_{x}\left(\xi, x_{n}\right), g_{y}\left(\xi, x_{n}\right)\right.$

,$\left.- g_{z}\left(\xi, x_{n}\right)\right)$ in the spectral domain, we define:

$$
\begin{aligned}
& g_{+}\left(\xi, x_{n}\right)=\frac{g_{x}\left(\xi, x_{n}\right)-i g_{y}\left(\xi, x_{n}\right)}{2}, \\
& g_{-}\left(\xi, x_{n}\right)=\frac{g_{x}\left(\xi, x_{n}\right)+i g_{y}\left(\xi, x_{n}\right)}{2},
\end{aligned}
$$

and

$$
\begin{aligned}
& \Pi_{+}^{\xi}\left(\mathbf{g}\left(\xi, x_{n}\right)\right):=\frac{\partial g_{+}\left(\xi, x_{n}\right)}{\partial z}+\frac{\xi}{2} g_{z}\left(\xi, x_{n}\right), \\
& \Pi_{-}^{\xi}\left(\mathbf{g}\left(\xi, x_{n}\right)\right):=\frac{\partial g_{-}\left(\xi, x_{n}\right)}{\partial z}-\frac{\xi}{2} g_{z}\left(\xi, x_{n}\right), \\
& \Pi_{z}^{\xi}\left(\mathbf{g}\left(\xi, x_{n}\right)\right)=\xi\left(g_{-}\left(\xi, x_{n}\right)+g_{+}\left(\xi, x_{n}\right)\right) .
\end{aligned}
$$


Using the Hankel representation given by Equation 43, and proper orthogonality properties of Bessel functions and integrability conditions, Equation 7 reduces to:

$$
b\left(\mathbf{F}^{q, m}, \mathbf{H}\right)=b\left(\mathbf{F}^{m}, \mathbf{H}^{m}\right)=b_{1}\left(\mathbf{F}^{m}, \mathbf{H}^{m}\right)-b_{2}\left(\mathbf{F}^{m}, \mathbf{H}^{m}\right),
$$

where

$$
\begin{aligned}
b_{1}\left(\mathbf{F}^{m}, \mathbf{H}^{m}\right) & =2\left\langle\Pi_{-}^{\xi_{q}}\left(\mathbf{F}^{m}\right), \tilde{\rho}_{\mathbf{t}} \Pi_{-}^{\xi_{q}}\left(\mathbf{H}^{m}\right)\right\rangle_{L^{2}} \\
& +2\left\langle\Pi_{+}^{\xi_{q}}\left(\mathbf{F}^{m}\right), \tilde{\rho}_{\mathbf{t}} \Pi_{+}^{\xi_{q}}\left(\mathbf{H}^{m}\right)\right\rangle_{L^{2}} \\
& +\left\langle\Pi_{z}^{\xi_{q}}\left(\mathbf{F}^{m}\right), \tilde{\rho}_{\mathbf{n}} \Pi_{z}^{\xi_{q}}\left(\mathbf{H}^{m}\right)\right\rangle_{L^{2}}, \\
b_{2}\left(\mathbf{F}^{m}, \mathbf{H}^{m}\right) & =i \omega \mu_{0}\left(2\left\langle F_{-}^{m}, H_{-}^{m}\right\rangle_{L^{2}}+2\left\langle F_{+}^{m}, H_{+}^{m}\right\rangle_{L^{2}}\right. \\
& \left.+\left\langle F_{z}^{m}, H_{z}^{m}\right\rangle_{L^{2}}\right) .
\end{aligned}
$$

and $\langle f, g\rangle_{L^{2}}=\int_{z} \bar{f} g d z$.

Using [47], the derivative of the magnetic field with respect to boundary position becomes:

$$
\begin{gathered}
\frac{d m}{d z_{i}}\left(z_{i}\right)=2\left[\tilde{\sigma}_{\mathbf{t}}\right]_{z_{i}}\left(\tilde{\rho}_{\mathbf{t}} \overline{\Pi_{-}^{\xi_{q}}\left(\mathbf{H}^{*, m}\right)} \tilde{\rho}_{\mathbf{t}} \Pi_{-}^{\xi_{q}}\left(\mathbf{H}^{m}\right)\right. \\
\left.+\tilde{\rho}_{\mathbf{t}} \overline{\Pi_{+}^{\xi_{q}}\left(\mathbf{H}^{*, m}\right)} \tilde{\rho}_{\mathbf{t}} \Pi_{+}^{\xi_{q}}\left(\mathbf{H}^{m}\right)\right)\left(z_{i}\right) \\
-\left[\tilde{\rho}_{\mathbf{n}}\right]_{z_{i}}\left(\overline{\Pi_{z}^{\xi_{q}}\left(\mathbf{H}^{*, m}\right)} \Pi_{z}^{\xi_{q}}\left(\mathbf{H}^{m}\right)\right)\left(z_{i}\right) .
\end{gathered}
$$

We use a fast inverse Hankel transform based on digital filters to transfer our solution to the space domain (see [27] for details)

\section{References}

1. Bakr, S.A., Pardo, D., Mannseth, T.: Domain decomposition Fourier FE method for the simulation of 3D marine CSEM measurements. Journal of Computational Physics 255, 456-470 (2013)

2. Bakr, S.A., Pardo, D.: A multi-domain decomposition-based Fourier finite element method for the simulation of 3D marine CSEM measurements. Computational Geosciences 21 (3), 345-357 (2017)

3. Key, K.: 1D inversion of multicomponent, multifrequency marine CSEM data: methodology and synthetic studies for resolving thin resistive layers. Geophysics 74 (2), F9-F20 (2009)

4. Puzyrev, V., Koldan, J., de la Puente, J., Houzeaux, G., Vázquez, M., MaríaCela, J.: A parallel finite element method for three-dimensional controlledsource electromagnetic forward modelling. Geophysical Journal International 193 (2), 678-693 (2013)

5. Alvarez-Aramberri, J., Pardo, D.: Dimensionally adaptive $h p$-finite element simulation and inversion of $2 \mathrm{D}$ magnetotelluric measurements. Journal of Computational Science 18, 95-105 (2017)

6. Martí, A.: The role of electrical anisotropy in magnetotelluric responses: from modelling and dimensionality analysis to inversion and interpretation. Surveys in Geophysics 35, 179-218 (2014)

7. Ijasan, O., Torres-Verdín, C., Preeg, W.E.: Inversion-based petrophysical interpretation of logging-while-drilling nuclear and resistivity measurements. Geophysics 78 (6), D473-D489 (2013) 
8. Pardo, D., Torres-Verdin, C.: Fast 1D inversion of logging-while-drilling resistivity measurements for the improved estimation of formation resistivity in high-angle and horizontal wells. Geophysics 80 (2), E111-E124 (2014)

9. Jia, Y., Wang, G.L., Abubakar, A.: Inversion for tilted triaxial conductivity in dipping layered formations. IEEE International Symposium on Antennas and Propagation \& USNC/URSI National Radio Science Meeting pp. $904-$ 905 (2015)

10. Rabinovich, M., Le, F., Lofts, J., S.Martakov: The vagaries and myths of look around deep resistivity measurements while drilling. Petrophysics 53 (2), 86-101 (2012)

11. Bittar, M., Klein, J., Beste, R., Hu, G., Wu, M., , Pitcher, J., Golla, C., Althoff, G., Sitka, M., Minosyan, V., Paulk, M.: A new azimuthal deep-reading resistivity tool for geosteering and advanced formation evaluation. SPE Reservoir Evaluation and Engineering 12 (2), 270-279 (2009)

12. Shahriari, M., Rojas, S., Pardo, D., Rodríguez-Rozas, A., Bakr, S.A., Calo, V.M., Muga, I.: A numerical 1.5D method for the rapid simulation of geophysical resistivity measurements. Geosciences 8(6), 1-28 (2018)

13. Davydycheva, S., Wang, T.: A fast modelling method to solve Maxwell's equations in 1D layered biaxial anisotropic medium. Geophysics 76 (5), F293-F302 (2011)

14. Loseth, L.O., Ursin, B.: Electromagnetic fields in planarly layered anisotropic media. Geophysical Journal International 170, 44-F80 (2007)

15. Tehrani, A.M., Slob, E.: Applicability of 1D and 2.5D marine controlled source electromagnetic modelling. Geophysical Prospecting 61, 602-613 (2013)

16. Plessix, R.E.: A review of the adjoint-state method for computing the gradient of a functional with geophysical applications. Geophysical Journal International 167(2), 495-503 (2006)

17. Natterer, F.: Adjoint methods as applied to inverse problems. In: Encyclopedia of Applied and Computational Mathematics, pp. 33-36. Springer Berlin Heidelberg (2015)

18. McGillivray, P., Oldenburg, D., Ellis, R., Habashy, T.: Calculation of sensitivities for the frequency-domain electromagnetic problem. Geophysics Journal International 116, 1-4 (1994)

19. Kong, J.A.: Electromagnetic wave theory. Wiley-Interscience (1986)

20. Kirsch, A., Hettlich, F.: The mathematical theory of time-harmonic Maxwell's equations, vol. 190. Springer, Switzerland (2015)

21. Ward, S.H., Hohmann, G.W.: Electromagnetic methods in applied geophysics: volume 1, theory. Society of Exploration Geophysicists (1987)

22. Dupuis, C., Denichou, J.M.: Automatic inversion of deep-directional-resistivity measurements for well placement and reservoir description. The Leading Edge 34(5), 50-512 (2015)

23. Demkowicz, L.: Finite element methods for Maxwell's equations. In: Encyclopedia of Computational Mechanics Second Edition, pp. 1-20. American Cancer Society (2017)

24. Pardo, D., Torres-Verdín, C., Paszynski, M.: Simulations of 3D DC borehole resistivity measurements with a goal-oriented $h p$ finite-element method. Part 2: through-casing resistivity instruments. Computational Geosciences 12(1), 83-89 (2008) 
25. Habashy, T., Anderson, B.: Reconciling differences in depth of investigation between $2-\mathrm{MHz}$ phase shift and attenuation resistivity measurements. Paper E presented at the 1991 SPWLA Annual Logging Symposium, Midland, Texas, 16-19 June (1991)

26. Desbrandes, R., Clayton, R.: Chapter 9 measurement while drilling. Developments in Petroleum Science 38, 251 - 279 (1994)

27. Key, K.: Is the fast Hankel transform faster than quadrature? Computer Physics Communications 77 (3), F21-F30 (2012) 